\title{
REFLECTION IN A CORNER FORMED BY THREE PLANE MIRRORS*
}

\author{
BY \\ J. L. SYNGE \\ The Ohio State University $\dagger$
}

1. Introduction. If a plane mirror is attached to the base of a projectile and a parallel beam of light projected on it, the direction of the reflected beam at any instant will give us information about the angular position of the projectile at that instant. It will not, however, indicate the angular position completely, because a rotation of the projectile about the normal to the mirror leaves the direction of the reflected ray unaltered.

This difficulty may be overcome by using more than one mirror, and the possibility of using a reflecting corner formed by three plane mirrors suggests itself. If the three mirrors are mutually perpendicular, the direction of the reflected beam gives no indication of the angular position of the projectile, because such a corner reverses the direction of any parallel beam falling on it. But if the angles of the corner are not right angles, this is no longer the case; in general, there will be six reflected beams, and their directions will determine the angular position of the projectile completely. With three parameters at our disposal (the three angles of the corner), we can secure a variety of different effects. The purpose of the present paper is to make a systematic study of the optical behavior of all corners formed by three plane mirrors.

The method used is based on the fact that the transformation of ray-directions due to reflection in a plane mirror is equivalent to a rigid-body rotation about the mirror-normal through two right angles (i.e. a half-turn), combined with a reversal of sense. Consequently, three successive reflections in three plane mirrors produce a transformation equivalent to three half-turns, combined with a reversal of sense. But, by Euler's theorem, three successive half-turns are equivalent to a single rotation (not, in general, itself a half-turn). Thus the transformation due to reflection in a corner formed by three plane mirrors may be described by giving the axis of the single equivalent rotation (called the optic axis in the present connection), and the angle of the rotation.

It is found that, when different orders of reflection in the three mirrors are taken in to consideration, there are in general three optic axes and a unique angle of rotation. The rotation occurs in both positive and negative senses, so that in general there are six reflected rays resulting from a given incident direction. This is, of course, to be expected, since we can form six permutations of three mirrors.

It is useful to represent directions by points on the surface of a unit sphere. There are then two fundamental spherical triangles. One has for vertices the normals to the three mirrors, and the other has for vertices three directed optic axes. Actually there are two spherical triangles formed by (undirected) optic axes, but one is the reflection of the other in the center of the sphere, and so we pick out one for definiteness. The

* Received Feb. 1, 1946. Ground.

† Part of this work was done by the author at the Ballistic Research Laboratory, Aberdeen Proving 
vertices of the triangle formed by the mirror-normals lie at the middle points of the sides of the triangle formed by the directed optic axes, and the angle of the single equivalent rotation is the defect from four right angles of the sum of the angles of the triangle formed by the directed optic axes.

Explicit formulae are given for the construction of the optic axes (3.10) and the angle of the single equivalent rotation (4.15).

The fact that a ray, to be reflected, must strike the front of a mirror, and not the back, introduces awkward conditions. These conditions are removed in the mathematical theory by supposing that the mirrors are planes which reflect from either side. Further, in investigating the effect of a second reflection, we may find that after reflection in the first mirror the course of the ray does not bring it in to incidence with the second mirror. In such cases we shall disregard the position of the ray, and apply to its'direction the transformation corresponding to reflection in the second mirror. These artificialities (from the practical standpoint) are introduced to avoid encumbering the mathematical theory with conditions which have no bearing on the fundamental transformation problem. Once the general theory has been set up, the conditions mentioned above may be looked in to in any particular case. To facilitate this, we shall continue to call one side of each mirror the front.

2. Equivalence of reflections and rigid body rotations. Let $\mathbf{N}$ be a unit vector normal to a plane mirror, drawn out from the front (Fig. 1). Let I be a unit vector along an incident ray, and $I^{\prime}$ a unit vector along the reflected ray. From a point $O$ let us drawn the unit vectors $\mathbf{N}, \mathbf{I}, \mathbf{I}^{\prime},-\mathbf{I},-\mathbf{I}^{\prime}$ (Fig. 2).

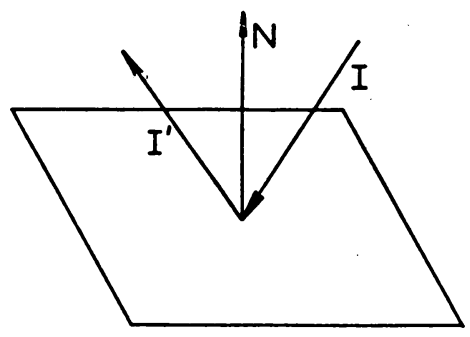

FIG. 1

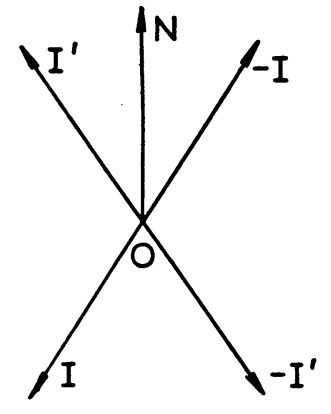

FIG. 2

It is clear from the law of reflection that $I^{\prime}$ is obtained from - I by a half-turn about $\mathbf{N}$; equivalently, $-\mathbf{I}^{\prime}$ is obtained from $\mathbf{I}$ by a half-turn about $\mathbf{N}$.

The transformations may also be shown on the surface of the unit sphere with center $O$; the unit vectors are now represented by points on the surface of the unit sphere (Fig. 3). The law of reflection may be stated as follows. Join $-\mathbf{I}$ to $\mathbf{N}$ by a great circle, and produce it on to make the arc $\left(\mathbf{N}, \mathbf{I}^{\prime}\right)$ equal to the $\operatorname{arc}(-\mathbf{I}, \mathbf{N})$. Equivalently, join $\mathbf{I}$ to $\mathbf{N}$ by a great circle, and produce it on to make the $\operatorname{arc}\left(\mathbf{N},-\mathbf{I}^{\prime}\right)$ equal to the arc $(\mathbf{I}, \mathbf{N})$.

In order that the incident ray may strike the front of the mirror, the $\operatorname{arc}(-\mathbf{I}, \mathbf{N})$ must be less than $\frac{1}{2} \pi$; but in view of the remarks made in Section 1, this restriction will not be imposed.

Consider now a corner formed by three plane mirrors with unit normals $\mathbf{N}_{\mathbf{1}}, \mathbf{N}_{\mathbf{2}}$, 
$\mathbf{N}_{3}$, drawn out from the fronts. These are represented on the unit sphere in Fig. 4. Consider successive reflections of an incident ray $\mathbf{I}$ in the order $\mathbf{N}_{1}, \mathbf{N}_{2}, \mathbf{N}_{3}$. We start by marking $-I$ on the unit sphere. We construct the first reflected ray $I^{\prime}$ by drawing
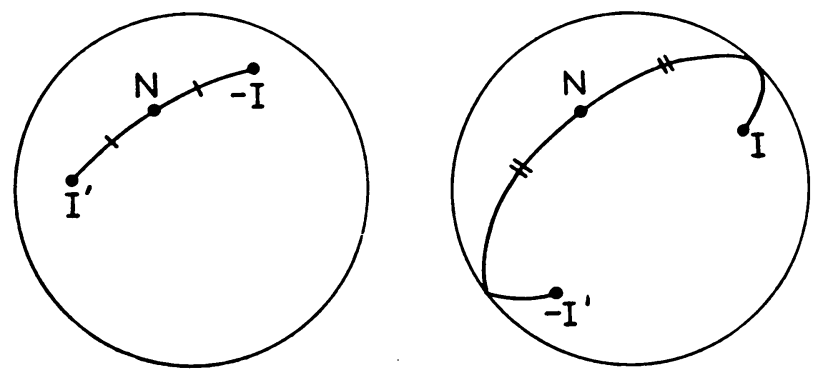

FIG. 3

a great circular arc from - I through $\mathbf{N}_{1}$, making $\mathbf{N}_{1}$ the point of bisection. If the second reflected ray is denoted by $I^{\prime \prime}$, we construct $-I^{\prime \prime}$ by drawing a great circular arc

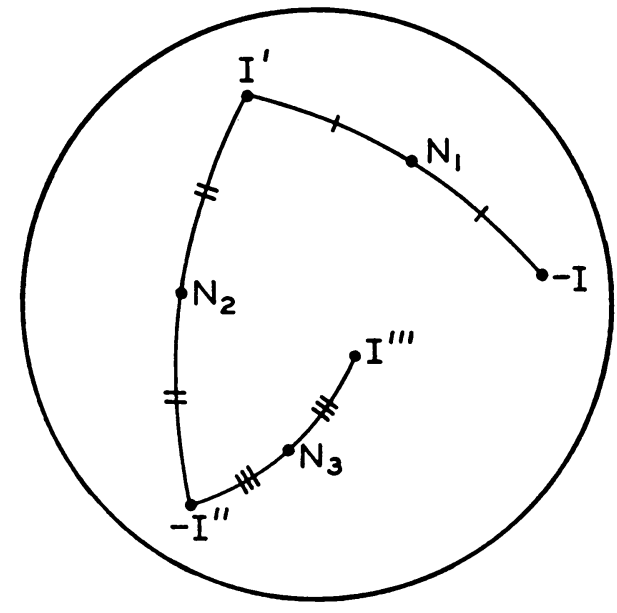

FIG. 4 from' $\mathbf{I}^{\prime}$ through $\mathbf{N}_{2}$, making $\mathbf{N}_{2}$ the point of bisection. We carry on from $-I^{\prime \prime}$ similarly through $\mathbf{N}_{3}$ to form the final ray $\mathbf{I}^{\prime \prime \prime}$.

We might also have started with $I$, instead of $-I$. The same rules of construction would have led us to $-I^{\prime \prime \prime}$.

If the reflections occur in a different order, we change the order of the points $\mathbf{N}_{1}, \mathbf{N}_{2}, \mathbf{N}_{\mathbf{3}}$ in the construction. In this way we get, in general, six different final directions $I^{\prime \prime \prime}$ corresponding to a single incident direction $\mathbf{I}$.

The transformations described above are equivalent to half-turns about diameters of a sphere, namely the diameters defined by $\mathbf{N}_{1}, \mathbf{N}_{2}, \mathbf{N}_{3}$, We know by Euler's theorem that any succession of rotations about diameters of a sphere is equivalent either tc no displacement at all or to a rotation through an angle less than $2 \pi$ about a uniquely determined diameter. The former alternative means that $I^{\prime \prime \prime}$ coincides with $-I$, no matter how $I$ is chosen. On the other hand, if there is a unique axis of equivalent rotation, then rays incident along the axis of that rotation (and such rays alone) will undergo reversal as a result of the triple reflection. Every other ray will undergo a change of direction determined by application of the equivalent rotation.

We shall call the axis of the equivalent rotation the undirected optic axis of the reflecting corner for the order of reflections assigned. This definition of optic axis would be adequate if we were content to have the term denote a diameter, without sense of direction. It is, however, desirable to understand by optic axis one of the two unit vectors lying on the axis of the equivalent rotation. Accordingly, we shall pro- 
ceed in the next section to define the directed optic axis. For the present, let us sum up our results as follows:

THEOREM I. For three successive reflections in a given order in three plane mirrors, either every incident ray is reversed in direction, or there exists a unique undirected optic axis such that

(a) a ray incident along the undirected optic axis is reversed in direction;

(b) the directions of reflected rays are obtained from the directions of incidents rays reversed by a rigid body rotation through an angle less than $2 \pi$ about the undirected optic axis.

3. Determination of the directed optic axes. Consider the following problem in spherical geometry: Given three points $\mathbf{N}_{\mathbf{1}}$, $\mathbf{N}_{2}, \mathbf{N}_{3}$ on a unit sphere, to construct a spherical triangle $\mathbf{A}_{1}, \mathbf{A}_{2}, \mathbf{A}_{3}$, such that $\mathbf{N}_{1}, \mathbf{N}_{2}, \mathbf{N}_{3}$ are the middle points of its sides (Fig. 5).

In vector notation, we have

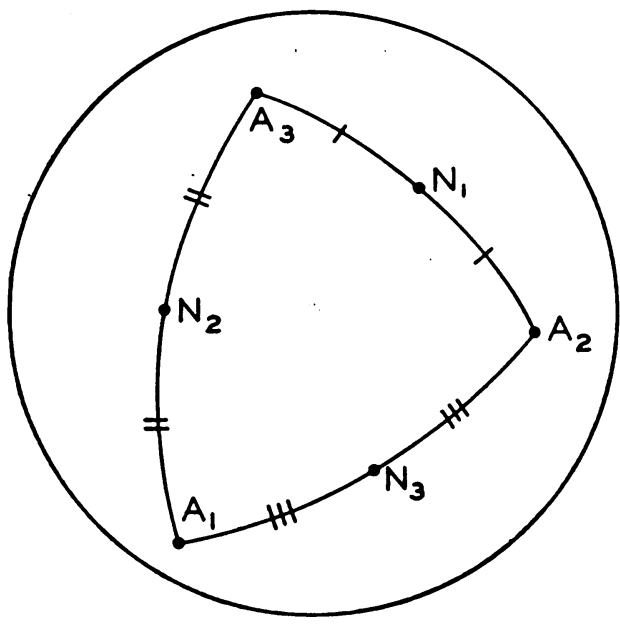

Fig. 5

$$
\mathbf{N}_{1} \cdot \mathbf{A}_{2}=\mathbf{N}_{1} \cdot \mathbf{A}_{3}, \quad \mathbf{N}_{2} \cdot \mathbf{A}_{3}=\mathbf{N}_{2} \cdot \mathbf{A}_{1}, \quad \mathbf{N}_{3} \cdot \mathbf{A}_{1}=\mathbf{N}_{3} \cdot \mathbf{A}_{2},
$$

and also

$$
L_{1} \mathbf{N}_{1}=A_{2}+A_{3}, \quad L_{2} \mathbf{N}_{2}=A_{3}+A_{1}, \quad L_{3} \mathbf{N}_{3}=A_{1}+A_{2},
$$

where the $L$ 's are unknown scalars. Our problem is to find the A's to satisfy (3.1) and (3.2).

Solving (3.2) for the A's, we get

$$
\begin{aligned}
& \dot{2 \mathbf{A}_{1}}=-L_{1} \mathbf{N}_{1}+L_{2} \mathbf{N}_{2}+L_{2} \mathbf{N}_{3}, \\
& 2 \mathrm{~A}_{2}=L_{1} \mathbf{N}_{1}-L_{2} \mathbf{N}_{2}+L_{3} \mathbf{N}_{3}, \\
& 2 \mathbf{A}_{3}=L_{1} \mathbf{N}_{1}+L_{2} \mathbf{N}_{2}-L_{3} \mathbf{N}_{3} .
\end{aligned}
$$

Let us define $M_{1}, M_{2}, M_{3}$ by

$$
M_{1}=\mathrm{N}_{2} \cdot \mathrm{N}_{3}, \quad M_{2}=\mathrm{N}_{3} \cdot \mathrm{N}_{1}, \quad M_{3}=\mathrm{N}_{1} \cdot \mathrm{N}_{2},
$$

these being of course the cosines of the angles between the $\mathbf{N}$ vectors. Taking the scalar products of the first two of (3.3) and $\mathbf{N}_{3}$, we get

$$
\begin{aligned}
& 2 \mathrm{~A}_{1} \cdot \mathbf{N}_{3}=-L_{1} M_{2}+L_{2} M_{1}+L_{3}, \\
& 2 \mathrm{~A}_{2} \cdot \mathbf{N}_{3}=L_{1} M_{2}-L_{2} M_{1}+L_{3} .
\end{aligned}
$$

Hence, by the last of (3.1),

$$
L_{1} M_{2}-L_{2} M_{1}=0
$$

Similarly

$$
L_{2} M_{3}-L_{3} M_{2}=0, \quad L_{3} M_{1}-L_{1} M_{3}=0 .
$$


If all the $M$ 's vanish (i.e. if the $\mathbf{N}$ vectors are mutually perpendicular), we may choose the $L$ 's arbitrarily, save for the condition that the sum of their squares shall equal 4. For it is easy to verify that the A's as given by (3.3) will then be unit vectors, and the conditions (3.1) and (3.2) will be satisfied. This means that we can take any point $A_{1}$ on the unit sphere and construct the triangle by passing successively through the $\mathbf{N}$ points, making each a point of bisection. The triangle necessarily closes.

Now suppose that there is at least one $M$ different from zero. From (3.6) and (3.7) it follows that a number $k$ exists such that

$$
L_{1}=2 k M_{1}, \quad L_{2}=2 k M_{2}, \quad L_{3}=2 k M_{3} .
$$

We proceed to determine $k$. Since the N's and the A's are unit vectors, if follows from (3.3) and (3.8) that

$$
k^{2}\left(M_{1}^{2}+M_{2}^{2}+M_{3}^{2}-2 M_{1} M_{2} M_{3}\right)=1 .
$$

The quantity in the parentheses is positive definite, since no $M$ can exceed unity in absolute value. We have then a choice between two real values of $k$, one negative and the other positive. If either of them be chosen, and the $L$ 's obtained from (3.8), and then the A's from (3.3), the conditions (3.1) and (3.2) are satisfied, and so the problem is solved. The problem then admits of two (and only two) solutions; the two triangles are the diametrical opposites of one another.

To avoid confusion, let us pick out one of these two solutions for application to the optical problem. Let us decide to take the positive value for $k$. To sum up, the triangle required is given by

$$
\begin{aligned}
& \mathbf{A}_{1}=k\left(-M_{1} \mathbf{N}_{1}+M_{2} \mathbf{N}_{2}+M_{3} \mathbf{N}_{3}\right), \\
& \mathbf{A}_{2}=k\left(\quad M_{1} \mathbf{N}_{1}-M_{2} \mathbf{N}_{2}+M_{3} \mathbf{N}_{3}\right), \\
& \mathbf{A}_{3}=k\left(\quad M_{1} \mathbf{N}_{1}+M_{2} \mathbf{N}_{2}-M_{3} \mathbf{N}_{3}\right),
\end{aligned}
$$

where the $M$ 's are given by (3.4) and

$$
k=\left(M_{1}^{2}+M_{2}^{2}+M_{3}^{2}-2 M_{1} M_{2} M_{3}\right)^{-1 / 2},
$$

the positive value being understood.

We have also

and

$$
\begin{aligned}
& \mathbf{N}_{1} \cdot \mathbf{A}_{2}=\mathbf{N}_{1} \cdot \mathbf{A}_{3}=k M_{1}, \\
& \mathbf{N}_{2} \cdot \mathbf{A}_{3}=\mathbf{N}_{2} \cdot \mathbf{A}_{1}=k M_{2}, \\
& \mathbf{N}_{3} \cdot \mathbf{A}_{1}=\mathbf{N}_{3} \cdot \mathbf{A}_{2}=k M_{3},
\end{aligned}
$$

$$
2 k M_{1} \mathbf{N}_{1}=\mathbf{A}_{2}+\mathbf{A}_{3}, \quad 2 k M_{2} \mathbf{N}_{2}=\mathbf{A}_{3}+\mathbf{A}_{1}, \quad 2 k M_{3} \mathbf{N}_{3}=\mathbf{A}_{1}+\mathbf{A}_{2} .
$$

We have now to show the connection of this problem in spherical geometry with our optical problem. We shall begin by proving that the diameter through $\mathbf{A}_{2}$ is the undirected optic axis for reflections in the order $\mathbf{N}_{1}, \mathbf{N}_{2}, \mathbf{N}_{3}$.

We take an incident ray with $\mathbf{I}= \pm \mathbf{A}_{\mathbf{2}}$. The first reflected ray is then given by $I^{\prime}=\mp A_{3}$. The second reflected ray is given by $I^{\prime \prime}= \pm A_{1}$. The third or final reflected ray is given by $\mathbf{I}^{\prime \prime \prime}=\mp \mathbf{A}_{\mathbf{2}}$. Thus $\mathbf{I}^{\prime \prime \prime}=-\mathrm{I}$, which proves that the diameter through $\mathbf{A}_{\mathbf{2}}$ is the undirected optic axis for reflections in the order $\mathbf{N}_{1}, \mathbf{N}_{2}, \mathbf{N}_{3}$. In just the same 
way we may show that the diameter through $\mathbf{A}_{2}$ is the undirected optic axis for reflections in the order $\mathbf{N}_{3}, \mathbf{N}_{2}, \mathbf{N}_{1}$, also.

Further, if we start from $\mathbf{A}_{3}$ or $\mathbf{A}_{1}$, we can prove in the same way that the diameter through $\mathbf{A}_{3}$ is the undirected optic axis for reflections in either of the orders $\mathbf{N}_{2}, \mathbf{N}_{3}, \mathbf{N}_{1}$ or $\mathbf{N}_{1}, \mathbf{N}_{3}, \mathbf{N}_{2}$, while the diameter through $\mathbf{A}_{1}$ is the undirected optic axis for reflections in either of the orders $\mathbf{N}_{3}, \mathbf{N}_{1}, \mathbf{N}_{2}$, or $\mathbf{N}_{2}, \mathbf{N}_{1}, \mathbf{N}_{3}$.

We are now in a position to define the directed optic axes by selecting senses on the undirected optic axes. We shall do this by imposing the condition that the directed optic axes are given by (3.10) with $k$ positive.

Let us sum up as follows:

THEOREM II. For any reflecting corner composed of three mirrors which are not all mutually perpendicular, the directed optic axes are the three unit vectors $\mathbf{A}_{1}, \mathbf{A}_{2}, \mathbf{A}_{3}$, given by (3.10) with positive $k$. The mirror-normals $\mathbf{N}_{1}, \mathbf{N}_{2}, \mathbf{N}_{3}$ meet the unit sphere at the middle points of the sides of the spherical triangle formed by $\mathbf{A}_{1}, \mathbf{A}_{2}, \mathbf{A}_{3}, \mathbf{N}_{1}$ being on the side $\mathbf{A}_{2} \mathbf{A}_{3}$, and so on. The directed optic optic axes correspond to the following orders of reflection:
Directed optic axis
Order of reflection
$\mathbf{A}_{1}$
$\mathbf{A}_{2}$
$\mathbf{N}_{3} \mathbf{N}_{1} \mathbf{N}_{2}$ or $\mathbf{N}_{2} \mathbf{N}_{1} \mathbf{N}_{3}$
$\mathbf{A}_{3}$
$\mathbf{N}_{1} \mathbf{N}_{2} \mathrm{~N}_{3}$ or $\mathrm{N}_{3} \mathrm{~N}_{2} \mathrm{~N}_{1}$
$\mathbf{N}_{2} \mathbf{N}_{3} \mathbf{N}_{1}$ or $\mathbf{N}_{1} \mathbf{N}_{3} \mathbf{N}_{2}$

We see from (3.10) that the directed optic axes are easily constructed in space by adding and subtracting the vectors $k M_{1} \mathbf{N}_{1}, k M_{2} \mathbf{N}_{2}, k M_{3} \mathbf{N}_{3}$. This construction is shown

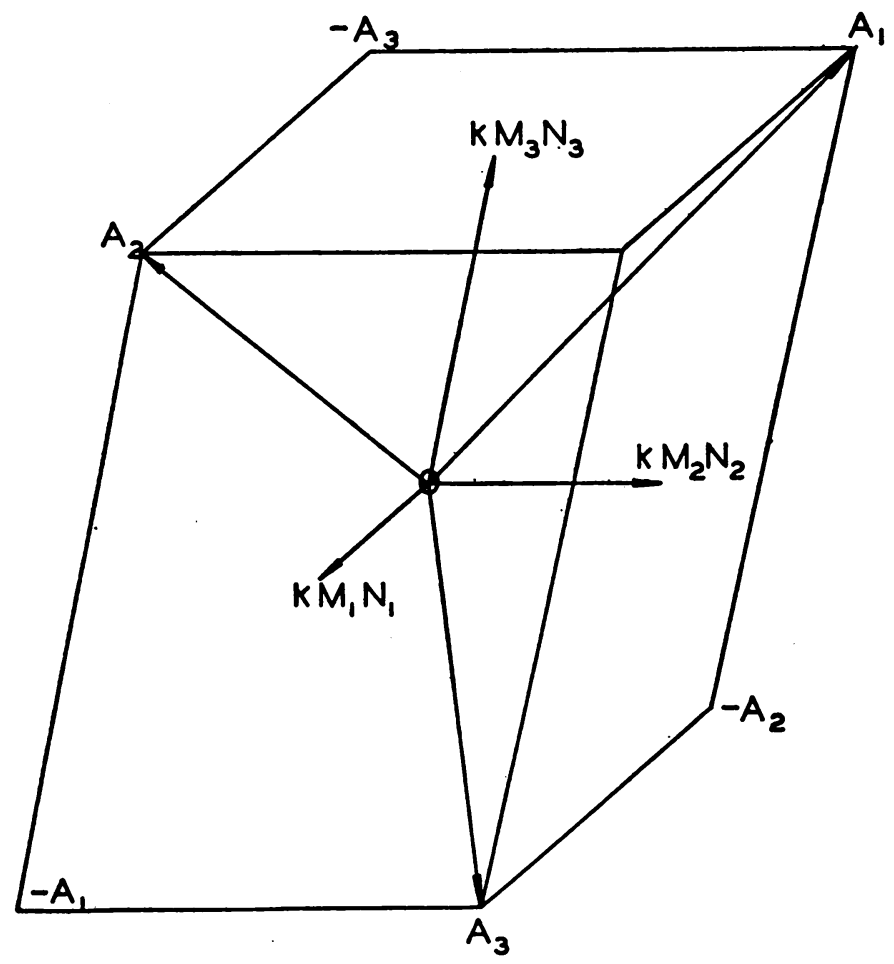

Fig. 6 
in Fig. 6. It is interesting to note that six of the eight vertices of the parallelepiped are occupied by the vectors $\pm A_{1}, \pm A_{2}, \pm A_{3}$.

4. The angle of the equivalent rigid-body rotation. Fig. 7 shows the representations on the unit sphere of the three mirror-normals $\mathbf{N}_{\mathbf{1}}, \mathbf{N}_{\mathbf{2}}, \mathbf{N}_{\mathbf{3}}$ and the three directed optic axes $\mathbf{A}_{1}, \mathbf{A}_{2}, \mathbf{A}_{\mathbf{3}}$. We know that three successive half-turns about $\mathbf{N}_{\mathbf{1}}, \mathbf{N}_{\mathbf{2}}, \mathbf{N}_{\mathbf{3}}$ in order are equivalent to a rotation about $\mathbf{A}_{2}$. We proceed to find the magnitude of this rotation.

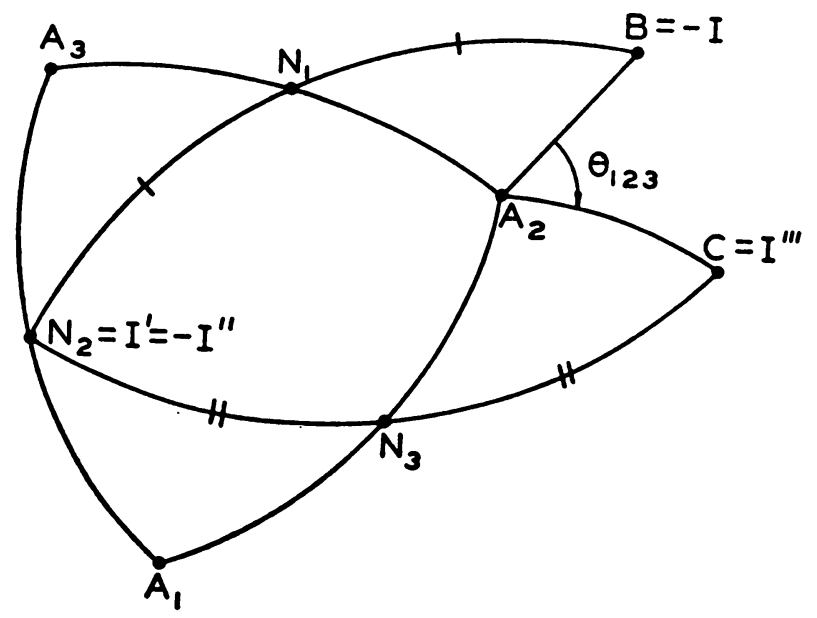

Fig. 7

We first construct the point $B$ by joining $\mathbf{N}_{2}$ to $\mathbf{N}_{1}$, and producing the great circle so as to make the arc $\left(\mathbf{N}_{1}, \mathbf{B}\right)$ equal to the $\operatorname{arc}\left(\mathbf{N}_{2}, \mathbf{N}_{1}\right)$. We then investigate the displacement of $B$ resulting from the three successive half-turns. It is clear that the final position $\mathbf{C}$ is obtained by joining $\mathbf{N}_{\mathbf{2}}$ to $\mathbf{N}_{\mathbf{3}}$, and producing the great circle so as to make the arc $\left(\mathbf{N}_{3}, \mathbf{C}\right)$ equal to the arc $\left(\mathbf{N}_{2}, \mathbf{N}_{3}\right)$. The effect of the three successive half-turns is to rotate the arc $\left(\mathbf{A}_{\mathbf{2}}, \mathbf{B}\right)$ into the arc $\left(\mathbf{A}_{\mathbf{2}}, \mathbf{C}\right)$. Since certain spherical triangles are obviously congruent, it is at once seen that the angle of rotation (taken less than $\pi$ ) is equal to the defect from four right angles of the sum of the angles of the spherical triangle $\mathbf{A}_{1} \mathbf{A}_{2} \mathbf{A}_{3}$. If the half-turns were carried out in the reverse order, then $\mathbf{C}$ would go to $B$; the angle of rotation would be the same in magnitude, but would have the opposite sense.

If the other senses of application of the three half-turns are considered, we get the same magnitude for the angle of the single equivalent rotation in all cases.

We shall now obtain a formula for the angle of the single equivalent rotation in terms of the angles between the normals of the three mirrors.

If an incident ray $I$ is reflected in a mirror with normal $N$, the reflected ray $I^{\prime}$ is given by

$$
\mathbf{I}^{\prime}=\mathbf{I}-2 \mathbf{N}(\mathbf{N} \cdot \mathbf{I}) \text {. }
$$

Let us start with an incident ray $I$ such that $-I=B$, where $B$ is as in Fig. 7, and let us follow this ray through successive reflections in the mirrors with normals $\mathbf{N}_{\mathbf{1}}, \mathbf{N}_{\mathbf{2}}, \mathbf{N}_{\mathbf{3}}$. The first reflected ray is $I^{\prime}=\mathbf{N}_{2}$, and so by (4.1)

$$
\mathrm{I}=\mathbf{I}^{\prime}-2 \mathbf{N}_{1}\left(\mathbf{N}_{1} \cdot \mathbf{I}^{\prime}\right)=\mathbf{N}_{2}-2 M_{3} \mathbf{N}_{1} \text {. }
$$


The second reflected ray is $I^{\prime \prime}=-\mathbf{N}_{2}$. The third and final reflected ray is

$$
\mathbf{I}^{\prime \prime \prime}=\mathbf{I}^{\prime \prime}-2 \mathbf{N}_{3}\left(\mathbf{N}_{3} \cdot \mathbf{I}^{\prime \prime}\right)=-\mathbf{N}_{2}+2 \mathbf{N}_{3}\left(\mathbf{N}_{3} \cdot \mathbf{N}_{2}\right)=-\mathbf{N}_{2}+2 M_{1} \mathbf{N}_{3},
$$

the $M$ 's being as in (3.4).

We note that (3.10) give

$$
-\mathrm{I} \cdot \mathbf{A}_{2}=\mathrm{I}^{\prime \prime \prime} \cdot \mathbf{A}_{2}=k M_{2} \text {. }
$$

As already seen, the angle of rotation $\theta_{123}$ is the angle between the $\operatorname{arcs}\left(A_{2}, B\right)$ and $\left(A_{2}, C\right)$, as shown in Fig. 7. This is the same as the angle between the vectors $\left(A_{2} \times B\right)$ and $\left(A_{2} \times C\right)$. If the rotation is considered positive when it is a right-handed rotation about $A_{2}$, through an angle less than $\pi$, then the angle satisfies the equation

$$
\sin \theta_{123} \cdot \mathbf{A}_{2}=\frac{\left(\mathbf{A}_{2} \times \mathbf{B}\right) \times\left(\mathbf{A}_{2} \times \mathbf{C}\right)}{\left|\mathbf{A}_{2} \times \mathbf{B}\right|\left|\mathbf{A}_{2} \times \mathbf{C}\right|},
$$

where $B=-I$ and $C=I^{\prime \prime \prime}$. (The angle $\theta_{123}$ shown in Fig. 7 is negative in the defined sense.)

To evaluate the right-hand side of (4.5), we note that by (4.4) we have

$$
\left|\mathrm{A}_{2} \times \mathrm{B}\right|\left|\mathrm{A}_{2} \times \mathrm{C}\right|=1-k^{2} M_{2}^{2} \text {. }
$$

Also, identically,

$$
\left(A_{2} \times B\right) \times\left(A_{2} \times C\right)=A_{2}\left[A_{2} \cdot(B \times C)\right] .
$$

By (4.2) and (4.3),

$$
\begin{aligned}
\mathrm{B} \times \mathrm{C} & =-\mathrm{I} \times \mathrm{I}^{\prime \prime \prime}=\left(-\mathrm{N}_{2}+2 M_{3} \mathbf{N}_{1}\right) \times\left(-\mathbf{N}_{2}+2 M_{1} \mathbf{N}_{3}\right) \\
& =-2 M_{1}\left(\mathbf{N}_{2} \times \mathbf{N}_{3}\right)-2 M_{3}\left(\mathbf{N}_{1} \times \mathbf{N}_{2}\right)-4 M_{1} M_{3}\left(\mathbf{N}_{3} \times \mathbf{N}_{1}\right),
\end{aligned}
$$

and so, by (3.10),

where $P$ is defined by

$$
\begin{aligned}
\mathrm{A}_{2} \cdot(\mathrm{B} \times \mathrm{C}) & =k\left(M_{1} \mathrm{~N}_{1}-M_{2} \mathrm{~N}_{2}+M_{3} \mathrm{~N}_{3}\right) \cdot(\mathrm{B} \times \mathrm{C}) \\
& =k P\left(-2 M_{1}^{2}+4 M_{1} M_{2} M_{3}-2 M_{3}^{2}\right),
\end{aligned}
$$

$$
P=\mathbf{N}_{1} \cdot\left(\mathbf{N}_{2} \times \mathbf{N}_{3}\right) .
$$

When the value (3.11) for $k$ is used, (4.9) may be written

$$
\mathbf{A}_{2} \cdot(\mathbf{B} \times \mathbf{C})=-2 P k^{-1}\left(1-k^{2} M_{2}^{2}\right) \text {. }
$$

On substitution of this expression in (4.7), and then substitution from (4.6) and (4.7) in (4.5), we get

$$
\sin \theta_{123}=-2 P k^{-1} \text {. }
$$

We note that $P$ is the determinant formed from the direction cosines of the three normals to the mirrors, and

Hence

$$
P^{2}=\left|\begin{array}{lll}
1 & M_{3} & M_{2} \\
M_{3} & 1 & M_{1} \\
M_{2} & M_{1} & 1
\end{array}\right|=1-k^{-2}
$$

$$
P=\epsilon\left(1-k^{-2}\right)^{1 / 2},
$$


where $\epsilon$ is +1 or -1 according as the orientation of the triad $\mathbf{N}_{1}, \mathbf{N}_{2}, \mathbf{N}_{3}$ is positive or negative.

We may sum up as follows:

THEOREM III. The magnitude (taken less than $\pi$ ) of the angle of the single equivalent rotation is the defect from four right angles of the angle-sum of the spherical triangle on the unit sphere whose vertices represent the directed optic axes. If a positive angle corresponds to a positive (right-handed) rotation about the directed optic axis involved, the angle is given both in magnitude and sign by

$$
\sin \theta=-2 \epsilon k^{-1}\left(1-k^{-2}\right)^{1 / 2},
$$

where $k$ is given in terms of the cosines of the angles between the mirror-normals by (3.11), and $\epsilon$ is +1 or -1 according as the triad of mirror-normals, in the order of the reflections, is positive or negative (i.e. right-handed or left-handed).

Let us now see how the six reflected rays are to be constructed when the incident ray $I$ is given and the three directed optic axes are known. We mark on the unit sphere (Fig. 8) the directed optic axes $\mathbf{A}_{1}, \mathbf{A}_{2}, \mathbf{A}_{3}$, and the incident ray reversed, $-\mathbf{I}$. Let us

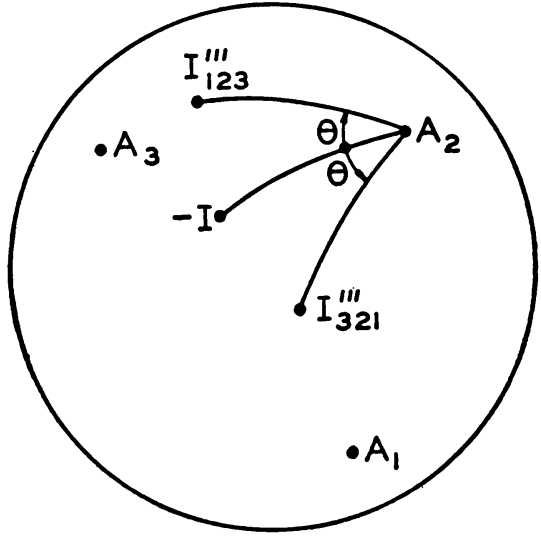

FIG. 8

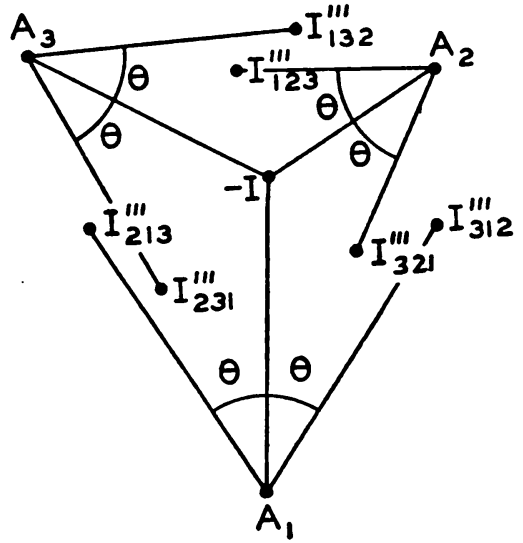

FIG. 9

suppose that the mirror-normals are so numbered that $\mathbf{N}_{\mathbf{1}}, \mathbf{N}_{\mathbf{2}}, \mathbf{N}_{\mathbf{3}}$ form a positive triad. To obtain the reflected ray resulting from reflections in the order $\mathbf{N}_{\mathbf{1}}, \mathbf{N}_{\mathbf{2}}, \mathbf{N}_{\mathbf{3}}$ we use the optic axis $\mathbf{A}_{2}$. We draw the arc $\left(\mathbf{A}_{2},-\mathbf{I}\right)$, and rotate it about $\mathbf{A}_{2}$ in the negative sense through an angle equal to the defect from four right angles of the angle-sum of the spherical triangle $\mathrm{A}_{1} \mathrm{~A}_{2} \mathrm{~A}_{3}$, or equivalently through an angle $\sin ^{-1} 2 k^{-1}\left(1-k^{-2}\right)^{1 / 2}$, as given in (4.15). This gives the reflected ray $I_{123}^{\prime \prime \prime}$, the subscripts indicating the order of the reflections. To obtain $\mathrm{I}_{321}^{\prime \prime}$, we rotate the arc $\left(\mathbf{A}_{2},-\mathbf{I}\right)$ about $\mathbf{A}_{2}$ in the opposite sense through the same angle.

Using the other optic axes, we obtain similarly the whole set of six reflected rays. These are shown in Fig. 9, the great circular arcs being shown as straight lines for simplicity. All the marked angles are equal. 
5. Cases of perpendicular mirrors. Let us consider the case where two of the mirrors are perpendicular to one another. Let us take $\mathbf{N}_{1}$ perpendicular to $\mathbf{N}_{2}$, and write

$$
M_{1} \neq 0, \quad M_{2} \neq 0, \quad M_{3}=0 .
$$

Then (3.10) give for the directed optic axes

and from (3.11) we have

$$
\begin{aligned}
& \mathbf{A}_{1}=k\left(-M_{1} \mathbf{N}_{1}+M_{2} \mathbf{N}_{2}\right), \\
& \mathbf{A}_{2}=k\left(\quad M_{1} \mathbf{N}_{1}-M_{2} \mathbf{N}_{2}\right), \\
& \mathbf{A}_{3}=k\left(\quad M_{1} \mathbf{N}_{1}+M_{2} \mathbf{N}_{2}\right),
\end{aligned}
$$

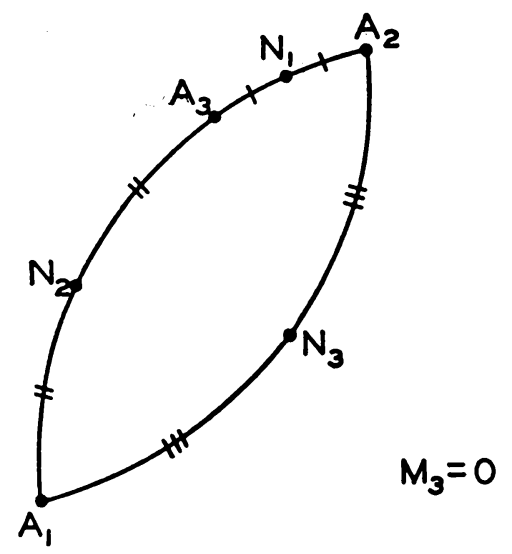

FIG. 10

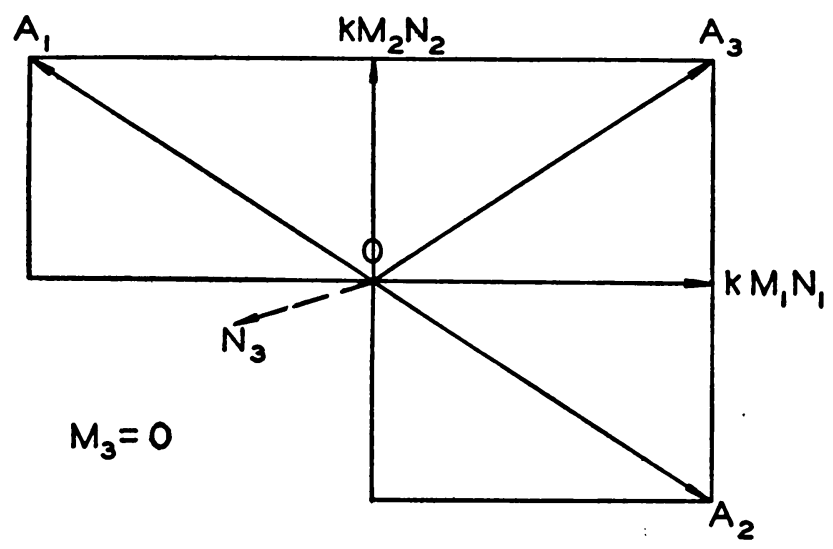

Fig. 11

$$
k=\left(M_{1}^{2}+M_{2}^{2}\right)^{-1 / 2} .
$$

The equations (3.12) and (3.13) become

$$
\mathbf{N}_{1} \cdot \mathbf{A}_{2}=\mathbf{N}_{1} \cdot \mathbf{A}_{3}=k M_{1}, \quad \mathbf{N}_{2} \cdot \mathbf{A}_{3}=\mathbf{N}_{2} \cdot \mathbf{A}_{1}=k M_{2}, \quad \mathbf{N}_{3} \cdot \mathbf{A}_{1}=\mathbf{N}_{3} \cdot \mathbf{A}_{2}=0,
$$


and

$$
\mathbf{A}_{2}+\mathbf{A}_{3}=2 k M_{1} \mathbf{N}_{1}, \quad \mathbf{A}_{3}+\mathbf{A}_{1}=2 k M_{2} \mathbf{N}_{2}, \quad \mathbf{A}_{1}+\mathbf{A}_{2}=0 .
$$

We note that $\mathbf{A}_{1}$ and $\mathbf{A}_{2}$ are opposed to one another, and $\mathbf{A}_{1}, \mathbf{A}_{2}, \mathbf{A}_{3}, \mathbf{N}_{1}, \mathbf{N}_{2}$ are coplanar. The arrangement on the unit sphere is shown in Fig. 10. The construction in space for the directed optic axes is shown in Fig. 11.

Let us now consider the case where one of the mirrors is perpendicular to the two others. Let $\mathbf{N}_{\mathbf{1}}$ be perpendicular to $\mathbf{N}_{\mathbf{2}}$ and $\mathbf{N}_{3}$. We write

$$
M_{1} \neq 0, \quad M_{2}=0, \quad M_{3}=0 .
$$

From (3.10) we get for the directed optic axes

$$
\mathbf{A}_{1}=-k M_{1} \mathbf{N}_{1}, \quad \mathbf{A}_{2}=k M_{1} \mathbf{N}_{1}, \quad \mathbf{A}_{3}=k M_{1} \mathbf{N}_{1},
$$

where $k=\left|M_{1}\right|^{-1}$; from (3.12) and (3.13)

$$
\mathbf{N}_{1} \cdot \mathbf{A}_{2}=\mathbf{N}_{1} \cdot \mathbf{A}_{3}=k M_{1}, \quad \mathbf{N}_{2} \cdot \mathbf{A}_{3}=\mathbf{N}_{2} \cdot \mathbf{A}_{1}=\mathbf{N}_{3} \cdot \mathbf{A}_{1}=\mathbf{N}_{3} \cdot \mathbf{A}_{2}=0,
$$

and

$$
\mathbf{A}_{2}+\mathbf{A}_{3}=2 k M_{1} \mathbf{N}_{1}, \quad \mathbf{A}_{3}+\mathbf{A}_{1}=0, \quad \mathbf{A}_{1}+\mathbf{A}_{2}=0 .
$$

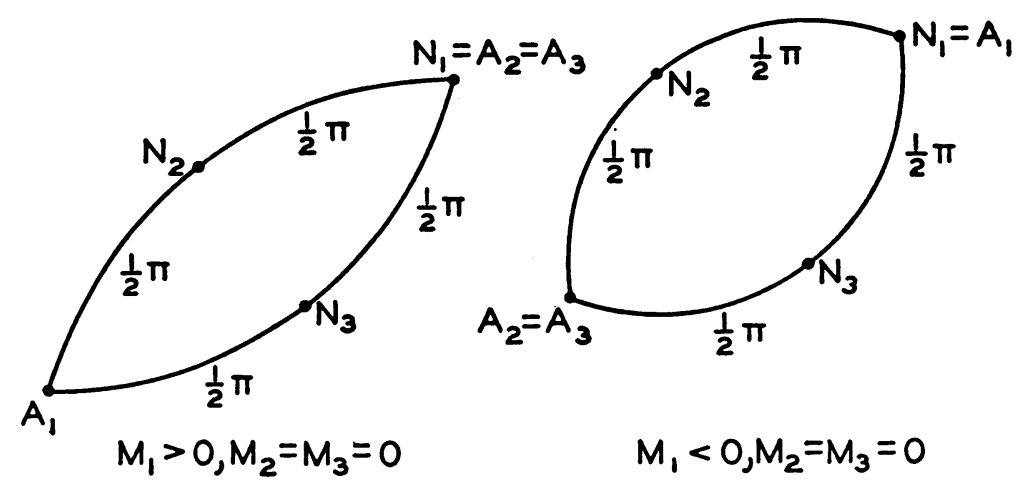

Fig. 12

Now $\mathbf{A}_{2}$ and $\mathbf{A}_{\mathbf{3}}$ coincide, and $\mathbf{A}_{\mathbf{1}}$ is opposed to them. The arrangement on the unit sphere is shown in Fig. 12 for the cases $M_{1}>0$ and $M_{1}<0$. 